\title{
ESQUEMA GERAL PARA ELUCIDAÇÃO DE SUBSTÂNCIAS ORGÂNICAS USANDO MÉTODOS ESPECTROSCÓPICO E ESPECTROMÉTRICO
}

\author{
Carlos Magno R. Ribeiro e Nelson Ângelo de Souza* \\ Departamento de Química Orgânica, Instituto de Química, Universidade Federal Fluminense, Outeiro de São João Batista, s/n, \\ 24020-150, Niterói - RJ, Brasil
}

Recebido em 21/8/06; aceito em 6/10/06; publicado na web em 28/5/07

\begin{abstract}
GENERAL SCHEME FOR ELUCIDATING THE STRUCTURE OF ORGANIC COMPOUNDS USING SPECTROSCOPIC AND SPECTROMETRIC METHODS. This work describes a systematic method to be applied in undergraduate courses of organic chemistry, correlating infrared spectra, hydrogen and carbon-13 nuclear magnetic resonance, and mass spectra. To this end, a scheme and a table were developed to conduct the elucidation of the structure of organic compounds initially using infrared spectra. Interpretation of hydrogen and carbon-13 nuclear magnetic resonance spectra and of mass spectra is used to confirm the proposed structure.
\end{abstract}

Keywords: infrared spectra interpretation; spectrometry spectra interpretation; organic compounds.

\section{INTRODUÇÃO}

As espectroscopias de infravermelho (IV), ressonância magnética nuclear de hidrogênio e carbono-13 (RMN de ${ }^{1} \mathrm{H}$ e $\left.{ }^{13} \mathrm{C}\right)$ e a espectrometria de massas (EM) são ferramentas atuais usadas para a elucidação estrutural de substâncias orgânicas. Devido a este fato, os cursos de graduação têm em sua grade curricular disciplinas que envolvem o ensino deste tema. Da mesma maneira, os livrostexto de química orgânica que são recomendados para estes cursos, de modo geral, introduzem a discussão sobre a análise estrutural de substâncias orgânicas, aplicando-se a interpretação de espectros de IV, RMN de ${ }^{1} \mathrm{H}$ e ${ }^{13} \mathrm{C}$, e massas ${ }^{1-4}$. Por outro lado, o tema análise estrutural que utiliza a espectroscopia e a espectrometria, atualmente, não faz parte da grade curricular dos ensinos fundamental e médio devido a sua especificidade. Assim, os alunos de graduação ao se depararem com estas técnicas apresentam certa dificuldade no aprendizado da análise dos espectros fornecidos pela espectroscopia de IV, RMN de ${ }^{1} \mathrm{H}$ e ${ }^{13} \mathrm{C}$ e EM.

O infravermelho é um método espectroscópico de análise que fornece informações quanto aos grupos funcionais que podem estar presentes na estrutura das substâncias. A ressonância magnética nuclear de hidrogênio e carbono-13 indica a disposição do esqueleto hidrocarbônico, ou seja, as diferentes situações de ambientes químicos dos átomos de hidrogênio e carbono na estrutura. A espectroscopia de massas, por sua vez, além de fornecer dados que permitam determinar a fórmula, pode contribuir na elucidação estrutural através dos padrões de fragmentação inerente às diferentes funções orgânicas.

Diversos livros didáticos ${ }^{1-4}$, como também os manuais ${ }^{5}$, ao discutirem este tema, apresentam uma série de tabelas de dados que visam facilitar a análise dos espectros das substâncias. Outros textos, além destas tabelas, apresentam esquemas ${ }^{6}$ que possam conduzir a elucidação estrutural através da interpretação de espectros de IV. Mais recentemente, um esquema para a interpretação de espectros de substâncias orgânicas também no IV foi desenvolvido por Lopes e Fascio ${ }^{7}$. Um fator que se deve mencionar é que os esquemas normalmente utilizados para ensino de elucidação estru-

*e-mail: gqoneas@vm.uff.br tural envolvem apenas o uso do IV, o qual não é um método completo, ou seja, na maioria das vezes o aluno não chega à elucidação da estrutura da substância, o que demonstra a necessidade de se recorrer a outros métodos espectrométricos. Por outro lado, outro fator importante no ensino do referido tema está na aplicação articulada destas metodologias de elucidação estrutural.

Devido ao nosso interesse no ensino de química ${ }^{8}$, resolvemos contribuir com um método que facilite a tarefa de elucidação estrutural de substâncias orgânicas tendo como ferramenta inicial a interpretação de espectros de IV, ao mesmo tempo em que se aplica a interpretação de espectros de ressonância magnética nuclear de hidrogênio e carbono-13 ( $\mathrm{RMN}$ de ${ }^{1} \mathrm{H}$ e ${ }^{13} \mathrm{C}$ ) e de massas para confirmar e/ou auxiliar na elucidação estrutural. Neste sentido, foram elaborados o Esquema 1 e a Tabela 1 resumida para conduzir o raciocínio do aluno na elucidação estrutural de substâncias orgânicas, demonstrando que as metodologias desenvolvidas devem e podem ser aplicadas em conjunto.

É válido aqui ressaltar que o Esquema 1 e a Tabela 1 apresentados neste artigo vêm sendo utilizados na disciplina Métodos Físicos de Análise, oferecida pelo Departamento de Química Orgânica (GQO) da Universidade Federal Fluminense (UFF). Esta disciplina integra a grade de disciplinas obrigatórias para os Cursos de Química Industrial e Bacharelado, assim como a grade de optativas para os cursos de Farmácia Industrial e Engenharia Química da UFF, além de outras disciplinas oferecidas pelo GQO.

\section{METODOLOGIA}

A interpretação de espectros de IV, RMN de ${ }^{1} \mathrm{H}$ e ${ }^{13} \mathrm{C}$, assim como de EM, envolve um "quebra-cabeça". Desta forma, informações que possam direcionar a resolução do problema devem ser obtidas previamente.

Duas possibilidades podem ser colocadas ao estudante para chegar à estrutura da substância a ser analisada: dispor apenas dos espectros de IV, RMN de ${ }^{1} \mathrm{H}$ e ${ }^{13} \mathrm{C}$ além de EM, logo, sem o conhecimento da fórmula molecular; envolver o conhecimento prévio da fórmula molecular da substância em questão.

Determinando a estrutura sem a fórmula molecular: neste caso, a análise inicia-se usando o Esquema 1, o qual fornecerá os núme- 


\section{Esquema de análise espectrométrica e espectroscópica de substâncias orgânicas (I.V.; RMN de ${ }^{1} \mathrm{H} \mathrm{e}^{13} \mathrm{C}$; Massas)}

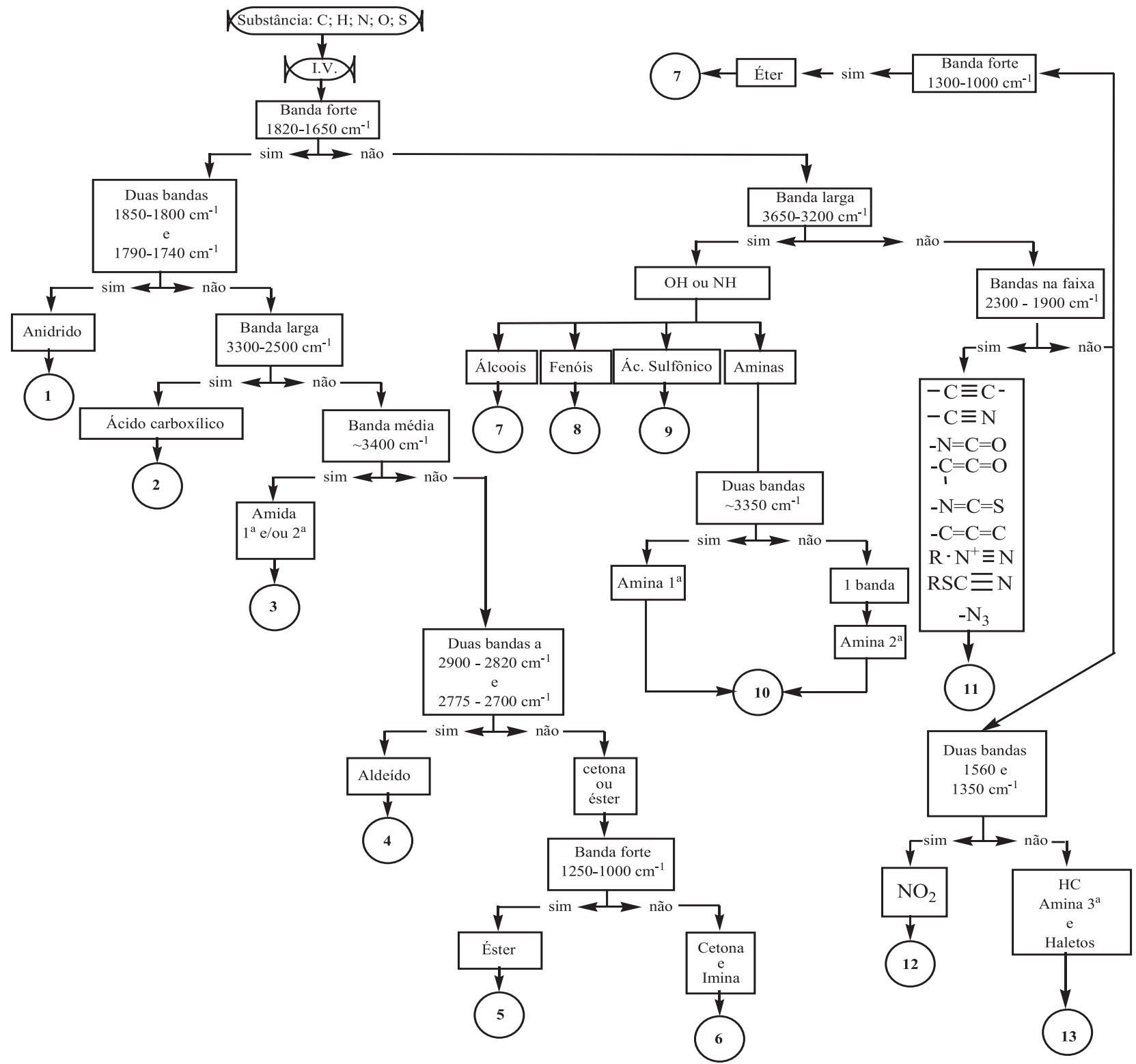

Esquema 1. Análise espectrométrica e espectroscópica de substâncias orgânicas (IV; RMN de ${ }^{1} \mathrm{H} e{ }^{13} \mathrm{C}$; Massas)

ros que devem ser usados na Tabela 1 para a seqüência da elucidação, bem como identificará a presença ou a ausência de átomos diferentes de carbono e hidrogênio na molécula, isto é, fornecerá as funções orgânicas presentes.

Assim, primeiramente devem ser respondidas as perguntas efetuadas no Esquema 1 até se alcançar os números que são apresentados no final de cada seqüência de perguntas. Neste primeiro momento, são obtidas informações sobre os grupos funcionais existentes na molécula. Caso a resposta inicial seja positiva, reinicia-se o processo seguindo o caminho que leva à primeira resposta negativa, na tentativa de verificar a possibilidade de outros grupos funcionais, uma vez que as substâncias podem ser polifuncionais. Em qualquer dos dois caminhos, cada vez que houver uma resposta positiva, devese recomeçar na resposta negativa correspondente, para averiguar a possibilidade da existência de outros grupos funcionais (substâncias polifuncionais). Logo, toda vez que se tem um não, não é necessário seguir o caminho do sim, porém, cada vez que se tem um sim, devese seguir o caminho correspondente para o não. Conhecendo-se, então, os elementos que podem estar presentes na substância, o seu peso molecular pode ser determinado através do perfil da região do íon molecular ( $\mathrm{M} ; \mathrm{M}+1 ; \mathrm{M}+2 ; \ldots)$ no EM. A seguir, aplica-se a regra dos $13^{9}$ para a dedução das bases das fórmulas moleculares. Equação geral: $\left[\mathrm{M}-\sum x(\mathrm{O})+y(\mathrm{~N})+z(\mathrm{~S})+w(\mathrm{X})\right] / 13=n+r / 13$; onde $x, y, z, n$ e $r$ são números inteiros; $\mathrm{O}$ é oxigênio, $\mathrm{N}$ é nitrogênio, $\mathrm{S}$ é enxofre e $\mathrm{X}$ é halogênio. Lembramos que nesta equação geral é possível acrescentar outros elementos químicos. Neste caso, basta acrescentar uma letra que represente um número inteiro e outra que represente o elemento identificado no IV. A base da fórmula molecular será $\mathrm{C}_{n} \mathrm{H}_{n+r}$. Exemplificando a construção da equação e da base da fórmula molecular: a) para uma substância contendo carbono, hidrogênio e oxigênio. Equação: $[\mathrm{M}-x(\mathrm{O})] / 13=n+r / 13$. Base da fórmula molecular $\mathrm{C}_{n} \mathrm{H}_{n+r} \mathrm{O}_{x}$; b) para uma substância contendo car- 
Tabela 1. Informações adicionais relevantes

\begin{tabular}{|c|c|c|c|c|}
\hline Item & $\begin{array}{c}\text { IV } \\
\text { Ligação: } \mathrm{cm}^{-1}\end{array}$ & $\begin{array}{c}\text { RMN de }{ }^{1} \mathrm{H} \\
\text { Ligação:ppm }\end{array}$ & $\begin{array}{l}\text { RMN de }{ }^{13} \mathrm{C}^{*} \\
\text { Ligação:ppm }\end{array}$ & EM \\
\hline 1 & - & - & $\mathbf{C}=\mathbf{O}: 155-180$ & - \\
\hline 2 & $\begin{array}{l}\text { C-O: } 1320-1210 \\
\text { O-H }(\delta \text { fora do plano) }: \sim 910 \\
\text { (banda triangular) }\end{array}$ & $\begin{array}{l}\text { RCOOH : } 11,0-12,00 \\
- \text { CH-COOH : } 2,1-2,5\end{array}$ & $\mathbf{C}=\mathbf{O}: 160-185$ & $\begin{array}{l}\mathrm{M}^{+\cdot}(\text { fraco }) \\
\mathrm{M}-17(\mathrm{M}-\mathrm{OH}) \\
\mathrm{M}-45(\mathrm{M}-\mathrm{COOH}) \\
\mathrm{m} / \mathrm{z}=60(\mathrm{RML})\end{array}$ \\
\hline 3 & $\begin{array}{l}\mathbf{C}=\mathbf{O}: 1680-1630(\text { Amida I) } \\
\mathbf{N}-\mathbf{H}: \delta 1640-1500 \text { (Amida II) }\end{array}$ & $\mathbf{N}-\mathbf{H}: 5-8$ & $\mathbf{C}=\mathbf{O}: 160-170$ & $\begin{array}{l}m / \mathrm{z}=44\left(\mathrm{CONH}_{2}\right)^{+} \\
m / \mathrm{z}=59 ; 73 ; 87 \ldots(\mathrm{RML})\end{array}$ \\
\hline 4 & - & -CㅌO : 9 - 10 & $\mathbf{C}=\mathbf{O}: 190-210$ & $\begin{array}{l}\mathbf{M}^{+} \text {(fraco para alifáticos; intenso para } \\
\text { aromáticos) } \\
\text { Alifáticos } \\
\mathbf{M}-29 \text { (perda de } \mathrm{CHO}) \\
\left.\mathrm{M}-43 \text { (perda de } \mathrm{H}_{2} \mathrm{C}=\mathrm{CHO}\right) \\
\mathrm{m} / \mathrm{z}=44 ; 58 ; 72 \ldots(\mathrm{RML}) \\
\text { Aromáticos } \\
\mathrm{M}-1 ; \mathrm{M}-29\end{array}$ \\
\hline
\end{tabular}

$5 \quad \mathbf{C}=\mathbf{O}: 1750-1740$

C-O : $1300-1050$

\author{
R-C \\ R-COO-CH : $3,5-4,8$
}

\section{Metílicos}

$\mathrm{M}^{+}$(fraco)

$\mathrm{M}-31\left(\mathrm{M}-\mathrm{OCH}_{3}\right)$

$m / \mathrm{z}=59\left(\mathrm{COOCH}_{3}\right)^{+}$

$m / z=74$ (RML)

Maiores

$\mathrm{M}-31 ; \mathrm{M}-45 ; \mathrm{M}-59$;

$\mathrm{M}-73 \ldots$ (perda de $-\mathrm{OR})$

$\mathrm{m} / \mathrm{z}=73 ; 87 ; 101(\mathrm{COOR})^{+}$

$m / \mathrm{z}=88 ; 102 ; 116 \ldots(\mathrm{RML})$
6 Cetonas alifáticas:

$\mathbf{C}=\mathbf{O}: 1720-1708$

Cetonas conjugadas:

C=O: $1700-1675$

Iminas: $\quad \mathbf{C}=\mathbf{N}: 1690-1640$
R-CH-COR : $2,1-2,4 \quad \mathbf{C}=\mathbf{O}: 190-210$ (singleto) $\quad \mathbf{M}^{+.}$(intenso)

Alifáticos

$\mathrm{M}-15 ; \mathrm{M}-29 ; \mathrm{M}-43 \ldots$

$m / \mathrm{z}=43 ; \mathrm{m} / \mathrm{z}=58 ; 72 ; 86 \ldots(\mathrm{RML})$

$\mathrm{m} / \mathrm{z}=42 ; 83$

Aromáticos

$m / \mathrm{z}=105 ; 120$

$\mathrm{M}^{+\cdot}$ (fraco ou ausente) $m / z=31 ; 45 ; 59 ; 73$..

$\mathrm{M}-18$ (perda de $\mathrm{H}_{2} \mathrm{O}$ )

R-CH $\mathrm{OH}_{2}$; RCHOHR'; RCOHR'R": $\mathrm{M}-\mathrm{R} ; \mathrm{M}-\mathrm{R}$; M-R” (clivagens $\alpha$ )

M-28; M-29

$\mathrm{m} / \mathrm{z}=\mathrm{M}-48$

$\mathrm{M} / \mathrm{M}+2: 100 / 4,4$

$\mathrm{M}^{+}$(fraco)

Regra do Nitrogênio

$m / \mathrm{z}=31 ; 44 ; 58 \ldots($ clivagem $\alpha)$

$\mathbf{C} \equiv \mathbf{N}$

$\mathrm{M}-1$

$m / z=41 ; 55 ; 69 \ldots($ RML $)$

$\mathrm{C}=\mathrm{C}$

$\mathrm{M}^{+}$(intenso) $\mathrm{M}-1$ (intenso)

$\mathrm{m} / \mathrm{z}=39($ clivagem $\beta$ )

-N=C=O: $2275-2250$

$\mathbf{- C}=\mathbf{C}=\mathbf{O}: 2150$

$-\mathbf{N}=\mathbf{C}=\mathbf{S}: 2140-1990$

$-\mathbf{C}=\mathbf{C}=\mathbf{C}: 1950$

$\mathbf{R}-\mathbf{N}^{+} \equiv \mathbf{N}: 2260$

$\mathbf{R S C} \equiv \mathbf{N}: 2175-2140$

$-\mathbf{N}_{3}: 2160-2120$ 
Tabela 1. (continuação)

\begin{tabular}{|c|c|c|c|c|}
\hline Item & $\begin{array}{c}\text { IV } \\
\text { Ligação: } \mathrm{cm}^{-1}\end{array}$ & $\begin{array}{c}\text { RMN de }{ }^{1} \mathrm{H} \\
\text { Ligação:ppm }\end{array}$ & $\begin{array}{l}\text { RMN de }{ }^{13} C^{*} \\
\text { Ligação:ppm }\end{array}$ & EM \\
\hline 12 & $\begin{array}{l}\text { nitroderivados alifáticos } \\
\mathrm{NO}_{2} \gamma_{\text {ass }}(\mathrm{F}) 1600-1530 \\
\gamma_{\mathrm{s}}(\mathrm{M}) 1390-1300 \\
\text { nitroderivados aromáticos } \\
\mathrm{NO}_{2} \gamma_{\text {ass }}(\mathrm{F}) 1550-1490 \\
\gamma_{\mathrm{s}}(\mathrm{F}) 1355-1315\end{array}$ & $-\mathbf{C H N O}_{2}: 4,1-4,3$ & 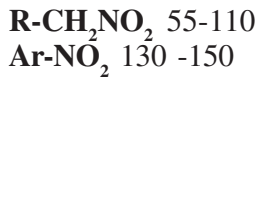 & $\begin{array}{l}\text { nitroderivados alifáticos: } \\
m / \mathrm{z}=30\left(\mathrm{NO}^{+}\right) \\
m / \mathrm{z}=46\left(\mathrm{NO}_{2}^{+}\right) \\
\mathrm{M}-\mathrm{NO}_{2} ; \\
\text { nitroderivados aromáticos: } \\
\mathrm{M}-\mathrm{NO} ; \mathrm{M}-\mathrm{NO}_{2}\end{array}$ \\
\hline 13 & $\begin{array}{l}\text { Alcanos } \\
\mathrm{C}-\mathrm{H}: \sim 3000 \\
\mathrm{CH}_{2}: \delta \sim 1465 ; 720 \text { (cadeia longa) } \\
\mathrm{CH}_{3}: \delta \sim 1375 \text { (muito fraco) } \\
\mathrm{C}-\mathrm{C}: \text { muito fraco } \\
\text { Alcenos } \\
=\mathrm{C}-\mathrm{H}: 3095-3010 \\
=\mathrm{C}-\mathrm{H} \delta \text { fora do plano 1000-650 } \\
\mathrm{C}=\mathrm{C}: 1660-1600 \\
\text { Aromáticos } \\
=\mathrm{C}-\mathrm{H}: 3050-3010 \\
=\mathrm{C}-\mathrm{H} \delta \text { fora do plano 900-690 } \\
\mathrm{C}=\mathrm{C}: 1600-1475 \\
\text { Haletos } \\
\mathrm{C}-\mathrm{F} 1400-1000 \\
\mathrm{C}-\mathrm{Cl} 800-600 \\
\text { C-Br } 750-500 \\
\text { C-I } 500\end{array}$ & 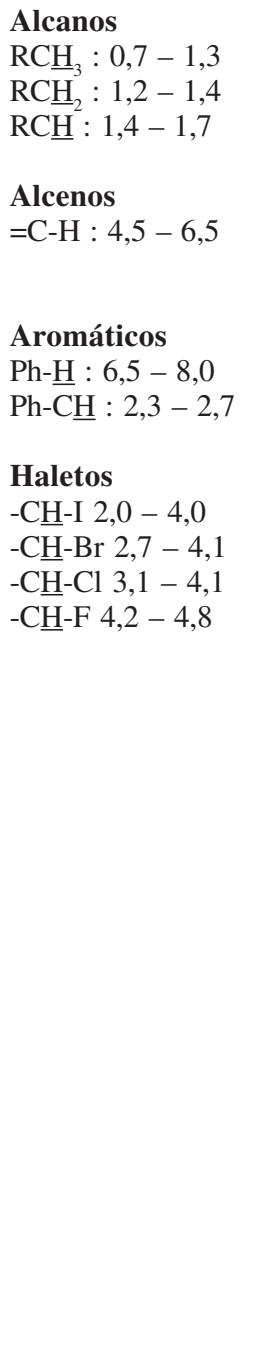 & $\begin{array}{l}\text { Alcanos } \\
\mathrm{CH}_{3}: 0-30 \\
\mathrm{CH}_{2}: 10-50 \\
\mathrm{CH}^{2}: 25-60 \\
\text { Alcenos } \\
\mathrm{C}=\mathrm{C}: 80-145\end{array}$ & 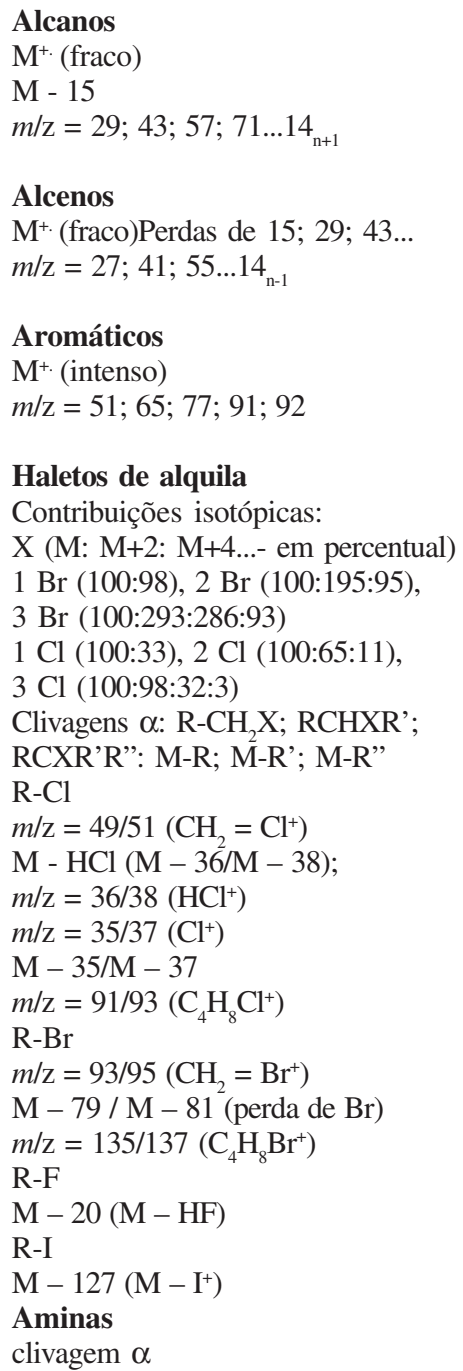 \\
\hline
\end{tabular}

* DEPT45 : $\mathrm{CH}(+) ; \mathrm{CH}_{2}(+) ; \mathrm{CH}_{3}(+) \quad$ DEPT90 : $\mathrm{CH}(+) ; \mathrm{CH}_{2}(0) ; \mathrm{CH}_{3}(0) \quad$ DEPT135 : $\mathrm{CH}(+) ; \mathrm{CH}_{2}(-) ; \mathrm{CH}_{3}(+)$

bono, hidrogênio e nitrogênio. Equação: $[\mathrm{M}-y(\mathrm{~N})] / 13=n+r / 13$. Base da fórmula molecular $\mathrm{C}_{n} \mathrm{H}_{n+r} \mathrm{~N}_{v}$; c) para uma substância contendo carbono, hidrogênio e enxofre. Equação: $[\mathrm{M}-z(\mathrm{~S})] / 13=n+r /$ 13; Base da fórmula molecular $\mathrm{C}_{n} \mathrm{H}_{n+r} \mathrm{~S}_{z}$. Após a determinação da base da fórmula molecular da substância em questão determina-se a fórmula molecular. Exemplificando: a) através do IV, identificou-se a existência da função ácido carboxílico $(\mathrm{RCOOH})$ e por meio do espectro de massas, o peso molecular de 88. Equação: [M - $x(\mathrm{O})] / 13$ $=n+r / 13$, substituindo os valores na equação, tem-se $[88-2(\mathrm{O})] / 13$ $=(88-32) / 13=56 / 13$; que é o mesmo que $4+4 / 13$ na regra dos 13 . Sabendo-se que a igualdade da equação geral é $n+r / 13$ e a base da fórmula geral é $\mathrm{C}_{n} \mathrm{H}_{n+r} \mathrm{O}_{x}$, tem-se a fórmula molecular $\mathrm{C}_{4} \mathrm{H}_{8} \mathrm{O}_{2}$.

Para se chegar à estrutura de uma substância a partir de sua fórmula molecular, calcula-se a seguir o índice de deficiência de hidrogênio (IDH), o qual indica a presença ou ausência de ligações duplas, triplas ou mesmo anéis na estrutura da substância em questão. Logo, o IDH corresponde ao número de mols de hidrogênio necessários para transformar uma substância insaturada em uma saturada e/ou uma substância cíclica em acíclica. Na literatura, encontram-se diversas maneiras para cálculo do $\mathrm{IDH}^{2,3}$, porém, neste artigo será aplicada a fórmula apresentada por Silverstein ${ }^{3}$ como ferramenta para obtenção do IDH: IDH $=\mathrm{C}+$ $1-\mathrm{H} / 2-\mathrm{X} / 2-\mathrm{N} / 2$; onde $\mathrm{C}$ representa o número de átomos de carbono, $\mathrm{H}$ o de hidrogênio, $\mathrm{X}$ o de halogênio e $\mathrm{N}$ o de nitrogênio. Exemplificando: uma substância com fórmula molecular $\mathrm{C}_{6} \mathrm{H}_{12}$ tem IDH igual a $1(\mathrm{IDH}=6+1-12 / 2=1)$. Uma substân- 
cia com fórmula molecular $\mathrm{C}_{6} \mathrm{H}_{5} \mathrm{NO}_{2}$ apresenta um IDH $=6+1$ $5 / 2+1 / 2=5$. No primeiro exemplo, com o valor de IDH igual a 1 , a estrutura da substância pode ser ciclo-hexano, 1-hexeno, 2hexeno, além de outras. No segundo caso, devido ao elevado grau de deficiência de hidrogênio, deve-se suspeitar da presença de sistema aromático ou poli-insaturado não aromático na substância, logo, a estrutura poderá ser nitrobenzeno.

Após o cálculo de IDH, deve-se rever o raciocínio usando o Esquema 1, conforme já mencionado, e a Tabela 1, que contemplam os métodos espectrais de IV, RMN de ${ }^{1} \mathrm{H} \mathrm{e}{ }^{13} \mathrm{C}$, assim como de EM.

Determinando a estrutura a partir da fórmula molecular: neste caso, a seqüência deve ser a mesma efetuada no item $a$ sem recorrer à regra dos 13, uma vez que a fórmula molecular já foi fornecida.

\section{ELUCIDANDO ESTRUTURAS DE SUBSTÂNCIAS ORGÂNICAS}

A seguir, serão apresentados dois exemplos para demonstrar a possibilidade da aplicação de forma articulada do Esquema 1 e da Tabela 1 desenvolvidos neste artigo. Estes exemplos servirão também para demonstrar a importância desta articulação ao se trabalhar estes diversos métodos espectrométricos de análise para melhor conduzir à elucidação da estrutura de uma determinada substância pelo aluno, o que seria mais difícil no caso de se utilizar apenas o IV. O raciocínio empregado nestes exemplos pode ser aplicado em qualquer outra substância no ensino deste tema.

\section{Determinando a estrutura de uma substância sem a fórmula molecular ${ }^{10}$}

Dados de IV: 2961(F); 2934(F); 2874(F); 1464(F); 1438; 1380; 1294; 1261(F); 1216(F); 741 e $644 \mathrm{~cm}^{-1}$. Dados de RMN de ${ }^{1} \mathrm{H}[\delta$ em ppm (multiplicidade, número de hidrogênio)]: 3,25(t, 2H); 2,11,1(m, 4H); 0,9(t, 3H). Dados de RMN de ${ }^{13} \mathrm{C}: 13,2 ; 21,4 ; 33,4 \mathrm{e}$ $34,9$ ppm. Dados de massas [ $\mathrm{m} / \mathrm{z}(\%)]: 26(6) ; 27(29) ; 28(13) ; 29(41)$; 38(2); 39(15); 40(22); 41(64); 42(3); 43(4); 50(2); 51(2); 53(2); 55(7); 56(16); 57(100); 58(5); 79(1); 81(1); 95(1); 93(1); 107(4); 109(4); 136(8); 138(8).

Inicia-se a análise usando-se o Esquema 1 e os dados de IV. Responde-se às perguntas contidas no Esquema 1, conforme discutido na metodologia. Através dos dados de IV, observa-se a ausência de banda forte na faixa de $1820-1650 \mathrm{~cm}^{-1}$, banda larga na faixa de $3650-3200 \mathrm{~cm}^{-1}$, bandas na faixa de $2300-1900 \mathrm{~cm}^{-1} \mathrm{e}$ bandas a 1560 e $1350 \mathrm{~cm}^{-1}$, o que leva à opção de número 13 no Esquema 1 . Neste caso, é possível a existência das funções hidrocarboneto (HC), amina terciária e haleto na estrutura molecular da substância em questão. A seguir, analisando-se o espectro de massas e observando-se o perfil da região do íon molecular, chega-se à presença de um átomo de bromo (136/138 u.m. na razão 1:1). Aplica-se a regra do 13. Equação: $[\mathrm{M}-w(\mathrm{X})] / 13$, substituindo os valores, tem-se [136 - 1x79]/13 =57/13; que é o mesmo que $4+5 / 13$ na regra dos 13 . Lembrando que a igualdade da equação geral é $n+r / 13$ e a base da fórmula geral é $\mathrm{C}_{n} \mathrm{H}_{n+r} \mathrm{Br}_{w}$, tem-se a fórmula molecular $\mathrm{C}_{4} \mathrm{H}_{9} \mathrm{Br}$. Após determinar a fórmula molecular, calcula-se o IDH. Aplicando-se a fórmula, obtém-se um valor igual a zero, o que significa ausência de insaturação e ciclos. Recorrendo-se às informações adicionais constantes da Tabela 1 item 13, com o espectro de massas, verifica-se um perfil de íon molecular com bromo, além dos fragmentos a 93/95 u.m. (íon $\mathrm{CH}_{2}=\mathrm{Br}^{+}$), indicando a presença de bromo no final da cadeia e a perda de $\mathrm{C}_{3} \mathrm{H}_{7}$ e do pico a 57 u.m. que indica a perda de bromo. Logo, confirma-se a saturação da molécula em questão e a presença de bromo na extremidade da cadeia. Analisando-se o espectro de RMN de ${ }^{1} \mathrm{H}$, constata-se a presença de três sinais: a) um tripleto a
3,25 ppm (indica hidrogênios ligados a um carbono que está ligado a um $\mathrm{CH}_{2}$ ), conforme a Tabela 1 item 13, este deslocamento corresponde a hidrogênio associado a um carbono que está ligado ao bromo, confirmando o dado fornecido pelo EM que aponta que o bromo está na extremidade da cadeia; b) um tripleto a 0,9 ppm (indica hidrogênios ligados a um carbono que está ligado a um $\mathrm{CH}_{2}$ ); c) um multipleto sobreposto entre 2,1-1,1 ppm. Analisando-se o espectro de $\mathrm{RMN}$ de ${ }^{13} \mathrm{C}$, observa-se a presença de quatro carbonos em ambientes químicos diferentes, descartando a possibilidade de ser um isômero ramificado. Estes dados conjugados entre si com a Tabela 1 item 10, com os dados fornecidos pelo Esquema 1 (hidrocarbonetos e haletos) e pelo IDH (ausência de insaturação) apontam para a fórmula estrutural 1-bromobutano.

\section{Determinando a estrutura de uma substância com fórmula molecular $\mathrm{C}_{4} \mathrm{H}_{9} \mathrm{Br}^{10}$}

Dados de IV: 2970(F); 2921(F); 2878(F); 2843; 1456(F); 1379; 1276; 1208(F); 1152(F); 993; 956; 842; 787 e $612 \mathrm{~cm}^{-1}$. Dados de RMN de ${ }^{1} \mathrm{H}$ [ $\delta$ em ppm (multiplicidade)]: 4,1(sexteto, $\left.1 \mathrm{H}\right) ; 1,9(\mathrm{~m}$, $2 \mathrm{H}) ; 1,8(\mathrm{~d}, 3 \mathrm{H})$ e $1,0(\mathrm{t}, 3 \mathrm{H})$. Dados de $\mathrm{RMN}$ de ${ }^{13} \mathrm{C}: 12,2 ; 26,0 ; 34,2$ e 53,4 ppm. Dados de massas [ $\mathrm{m} / \mathrm{z}(\%)]: 26(4) ; 27(19) ; 28(5) ; 29(41)$; 38(2); 39(14); 40(2); 41(53); 42(3); 50(2); 51(2); 53(2); 55(5); 56(8); 57(100); 58(4); 79(1); 81(1); 107(1); 109(1); 136(1); 138(1).

Inicialmente determina-se o IDH. Aplicando-se a fórmula, obtém-se um valor igual a zero, significando ausência de insaturação e ciclos. A seguir, responde-se às perguntas contidas no Esquema, conforme discutido na metodologia. Através dos dados de IV, observa-se a ausência de banda forte na faixa de $1820-1650 \mathrm{~cm}^{-1}$, banda larga na faixa de $3650-3200 \mathrm{~cm}^{-1}$, bandas na faixa de 2300 $1900 \mathrm{~cm}^{-1}$ e bandas a 1560 e $1350 \mathrm{~cm}^{-1}$, o que leva à opção de número 13 no Esquema 1. Neste caso, é possível a existência das funções hidrocarboneto (HC), amina terciária e haleto na estrutura molecular da substância em questão. Recorrendo-se às informações adicionais constantes da Tabela 1 item 13 com o espectro de massas, verifica-se um perfil de íon molecular com bromo, além dos fragmentos a 107/109 u.m. (íon $\mathrm{CH}_{3} \mathrm{CH}=\mathrm{Br}^{+}$) que indica a perda de $\mathrm{C}_{2} \mathrm{H}_{5}$ (29 u.m.) pela clivagem alfa, e do pico a 57 u.m. indicando a perda de bromo. Logo, confirma-se que a molécula em questão é saturada, a presença de bromo, conforme fórmula molecular fornecida, bem como a presença do grupo etila. Analisando-se o espectro de RMN de ${ }^{1} \mathrm{H}$, constata-se a presença de quatro sinais: um tripleto a 1,0 ppm (indica hidrogênios ligados a um carbono que está ligado a um $\mathrm{CH}_{2}$ ), um dubleto a 1,8 ppm (indica hidrogênios ligados a um carbono que está ligado a um $\mathrm{CH}$ ), um multipleto a 1,9 ppm, além de um sexteto a 4,1 ppm (região de hidrogênios ligados a carbonos com bromo - Tabela 1, item 13). Estes dados conjugados entre si com a Tabela 1, item 13, com os dados fornecidos pelo Esquema 1 (hidrocarbonetos e haletos) e pelo IDH (ausência de insaturação) apontam para a fórmula estrutural 2-bromobutano. Analisando-se o espectro de RMN de ${ }^{13} \mathrm{C}$, observa-se a presença de quatro carbonos em ambientes químicos diferentes, cujo pico a $55 \mathrm{ppm}$ é relativo a $\mathrm{CHBr}$ (conforme Tabela 1, item 13), descartando também a possibilidade de ser um isômero ramificado, confirmando a estrutura proposta.

\section{CONCLUSÕES}

Neste trabalho foi apresentado o Esquema 1 e a Tabela 1 resumida para conduzir o raciocínio do aluno na elucidação estrutural de substâncias orgânicas, demonstrando que as metodologias espectrométricas desenvolvidas para elucidação estrutural devem e podem ser aplicadas de maneira articulada no ensino deste tema. 


\section{AGRADECIMENTOS}

Ao Departamento de Química Orgânica da UFF pela colaboração na execução deste trabalho.

\section{REFERÊNCIAS}

1. Harwood, L. M.; Claridge, T. D. W.; Introduction to Organic Spectroscopy, $1^{\text {st }}$ ed., Oxford Science Publication: Great Britain, 2000.

2. Pavia, D. L.; Lampman, G. M.; Krij, J.; Introduction to Spectroscopy - A Guide for Students of Organic Chemistry, $1^{\text {st }}$ ed., Cruzda, Brooks: Cole Thonson Learning, 2001.

3. Silverstein, R. M.; Webster, F. X.; Identificação Espectrométrica de Compostos Orgânicos, 6ª ed., LTC: Rio de Janeiro, 2000.

4. Lambert, J. B.; Shurvell, H. F.; Ligntner, D. A.; Introduction to Organic Spectroscopy, $1^{\text {st }}$ ed., McMillan Publishing Company: New York, 1987.

5. Pretsch, E.; Clerc, T.; Seibl, J.; Simon, W.; Tablas para elucidacion structural de compuestos organicos por metodos espectroscopicos, 1르 ed., Editorial Alhambra S.A.: Espanha, 1980.
6. Wilcox Jr., C. F.; Experimental Organic Chemistry - A small-scale approach, $1^{\text {st }}$ ed., McMillan Publishing Company: New York, 1997, p. 103-202 e 486.

7. Lopes, W. A.; Fascio, M.; Quim. Nova 2004, 27, 670.

8. Soares, B. G.; Souza, N. A.; Pires, D. X.; Química Orgânica: Teoria e Técnicas de Preparação e Identificação de Compostos Orgânicos, 1aㅡ. ed., Editora Guanabara: Rio de Janeiro, 1988; Souza, N. A.; Coutinho, L. G. R. Em Contribuições aos Professores de Química do Ensino Médio; Coutinho, L. G. R.; Ferreira, V. F., eds.; Ed. UFF: Niterói, 2005, cap. 12; Coutinho, L. G. R.; Souza, N. A. Em Contribuições aos Professores de Química do Ensino Médio; L. G. R.; Ferreira, V. F., eds.; Ed. UFF: Niterói, 2005, cap. 13; Borges, M. N.; Ribeiro, C. M. R.; Souza, N. A.; Educ. Química 2005, 16, 586; Ribeiro, C. M. R. Em Contribuições aos Professores de Química do Ensino Médio; L. G. R.; Ferreira, V. F., eds.; Ed. UFF: Niterói, 2005, cap. 16; Souza, N. A.; Bravo, R. V. F.; J. Chem. Educ. 1989, 66, 1039; Soares, B. G.; Pires, D. X.; Souza, N. A.; Quim. Nova 1987, 10, 27.

9. Bright, J. W.; Chen, E. L. M.; J. Chem Educ.1983, 60, 557.

10. Os dados de IV desta substância foram obtidas em aparelho Spectrum One spectrometer; PerkinElmer Instruments da UFF. Os dados de RMN de ${ }^{1} \mathrm{H} \mathrm{e}$ ${ }^{13} \mathrm{C}$, bem como os dados de massas foram obtidos em http://www.aist.go.jp/ RIODB/chem.html, acessada em Julho 2006. 